\title{
Empleo y brecha digital de género en América Latina
}

Revista Latinoamericana de Economía y Sociedad Digital

Issue 1 , agosto 2020

Autores: Hernan Galperin(D), Malena Arcidiacono(D)

DOI: $10.53857 /$ GEPT8927

Publicado: 10 agosto, 2021

Cita sugerida: Galperín, Hernán \& Arcidiacono, Malena (2020) “Empleo y brecha digital de género en América Latina" en Revista Latinoamericana de Economía y Sociedad Digital, Issue 1

Licencia: Creative Commons Atribución-NoComercial 4.0 Internacional (CC BY-NC 4.0)

Tipo: Ensayo

Palabras clave: América Latina, Brecha digital, Empleo, Género, Uso de Internet

\section{Resumen}

Existe una amplia literatura que examina los determinantes de la brecha digital de género en los países en desarrollo y presenta recomendaciones de políticas para mitigarla. Sin embargo, pocos trabajos han estudiado cómo las diferencias de género en patrones de empleo afectan la adopción de TIC en general, y el uso de Internet en particular. Esto es importante porque la participación en la fuerza laboral y los tipos de trabajos que realizan hombres y mujeres a menudo se correlacionan con diferentes oportunidades para acceder a Internet y desarrollar habilidades TIC. Este estudio contribuye a llenar este vacío de conocimiento al explorar cómo las diferencias de género en el empleo afectan la brecha digital de género en tres países latinoamericanos (Ecuador, México y Perú). Los hallazgos identifican de manera concluyente el empleo como el factor que más contribuye a la brecha de uso de Internet entre hombres y mujeres, por delante de otros predictores tradicionales como la edad, la educación y los ingresos. Los resultados también sugieren que el estar ocupado afecta significativamente más el uso de Internet por parte de las mujeres que entre los hombres, lo que se atribuye al hecho de que las mujeres tienden a trabajar en sectores más intensivos en TIC (por ejemplo, servicios de salud y educación). Las estimaciones de límite inferior del análisis de descomposición indican que, si las mujeres estuvieran 
ocupadas a la misma tasa que los hombres, la brecha digital de género en estos países se reduciría en al menos una cuarta parte.

\section{Introducción}

A pesar del incremento sostenido en la conectividad a Internet en los países en desarrollo en las últimas décadas, los datos indican que persiste una considerable brecha de género en la adopción de Tecnologías de la Información y las Comunicaciones (TIC). A nivel global, la Unión Internacional de Telecomunicaciones (UIT) estima que la brecha de género en el uso de Internet en los países emergentes es de aproximadamente 12 puntos porcentuales (p.p.), pero la brecha es significativamente mayor en los países menos desarrollados, donde la tasa de conectividad entre mujeres alcanza solo la mitad de la tasa para los hombres (ITU 2019). Por otro lado, contrariamente a las expectativas, la brecha relativa en el uso de Internet entre hombres y mujeres está creciendo en los países en desarrollo, lo que contradice la suposición generalizada sobre la reducción en las diferencias de género en el acceso a Internet a lo largo del tiempo.

Existe una amplia literatura a nivel global que examina los determinantes de la brecha digital de género y presenta recomendaciones de política pública para mitigarla (Wasserman y Richmond-Abbott 2005, Alozie y Akpan-Obong 2017, Rashid 2016, Gray et al. 2017, Bhandari 2019). Sin embargo, muy pocos estudios examinan cómo las diferencias de género en los patrones de empleo afectan la adopción de TIC en general y el uso de Internet en particular. Esto es importante porque la participación en la fuerza laboral y los tipos de trabajos que realizan hombres y mujeres a menudo se correlacionan con diferentes oportunidades para acceder a Internet y desarrollar habilidades TIC. En el contexto de los países en desarrollo, el rol del uso de tecnología en el trabajo se amplifica por la penetración relativamente limitada de la banda ancha residencial.

Este estudio examina cómo las diferencias de género en los patrones de empleo afectan la brecha en el uso de Internet entre hombres y mujeres en Ecuador, México y Perú. Más específicamente, nuestra hipótesis sugiere que las diferencias de género en los patrones de empleo son un determinante central de la brecha digital de género. Por ejemplo, la menor participación laboral femenina y la mayor incidencia del trabajo a tiempo parcial entre mujeres están asociadas con menores oportunidades de acceder a Internet en el trabajo o desarrollar habilidades TIC a través de la capacitación laboral y el uso intensivo de Internet. $\mathrm{Al}$ mismo tiempo, es más probable que las mujeres trabajen en el sector de comercio minorista, salud, educación y otros sectores intensivos en TIC, lo que potencialmente aumenta el efecto del empleo en las habilidades TIC y en el uso de Internet.

Los datos para este estudio provienen de encuestas de hogares representativas a nivel nacional en Ecuador, México y Perú. Estas encuestas son administradas por las oficinas nacionales de estadística de cada país y contienen información limitada sobre el uso de 
Internet, que se refieren al tipo de actividades y al lugar más común de uso. Al mismo tiempo, el tamaño de la muestra permite estimaciones robustas utilizando técnicas estadísticas inviables con muestras más pequeñas. Para este estudio, nuestro análisis combina la regresión multivariada con una técnica de descomposición desarrollada por Fairlie (1999) para estimar la contribución de las diferencias en el empleo a la brecha digital de género en estos tres países latinoamericanos.

De modo general, los resultados indican que las diferencias de género en el empleo son las que contribuyen en mayor medida a la brecha de género en el uso de Internet, excepto en un modelo para Perú donde las diferencias de género en el empleo se encuentran apenas por debajo de las diferencias en el nivel educativo. Los resultados sugieren también que, controlando otras variables que afectan el uso de Internet, la participación laboral afecta significativamente más el uso de Internet entre las mujeres que entre los hombres, lo que atribuimos al hecho de que las mujeres tienden a trabajar en sectores más intensivos en TIC (por ejemplo, servicios de salud, educación, etc.). Si las mujeres estuvieran ocupadas a una tasa igual que los hombres, nuestras estimaciones de límite inferior sugieren que la brecha digital de género se reduciría al menos en una cuarta parte.

El presente estudio realiza dos contribuciones significativas al estudio tradicional de la brecha digital de género. En primer lugar, los resultados para los tres países estudiados arrojan luz sobre el nexo entre los patrones en el empleo (incluida la división del trabajo doméstico) y el uso de Internet. En segundo lugar, al mostrar la relevancia del factor empleo, el presente documento contribuye al diseño de políticas de conectividad orientadas a reducir las brechas de género en el uso de Internet y habilidades TIC. Pese a los numerosos avances logrados, dichas políticas siguen siendo relevantes para los países de la región, ya que en muchos de ellos el uso regular de Internet apenas alcanza a la mitad de la población adulta. Esto difiere de los países más avanzados donde el no uso de Internet está limitado a una fracción relativamente pequeña de la población adulta, y donde la paridad de género es significativamente mayor en el empleo, la educación y en otras áreas clave.

El trabajo se organiza de la siguiente manera. En la siguiente sección se presenta una revisión de la literatura sobre el nexo entre las diferencias de género en el empleo y la adopción de TIC, junto a una descripción general de la situación del empleo en los países en estudio que ayuda a contextualizar los hallazgos. Dicha sección también proporciona antecedentes teóricos para las principales preguntas de investigación abordadas en el estudio. En la sección 3 se discuten los métodos y variables, conjuntamente con la presentación de estadísticas descriptivas sobre el empleo y el uso de Internet. La presentación de resultados en la sección 4 es seguida por una discusión de los hallazgos y las conclusiones, incluyendo las implicancias para las iniciativas de conectividad en los países emergentes. 


\section{Antecedentes y teoría}

Múltiples indicadores provenientes de organismos multilaterales sugieren que persisten grandes disparidades de género en los mercados laborales de América Latina. En promedio, las mujeres reciben aproximadamente un $20 \%$ menos que los hombres que realizan un trabajo similar y con calificaciones similares. A pesar de los continuos avances en logros educativos, la participación femenina en la fuerza laboral se ha nivelado en aproximadamente 25 p.p. menos que la de los hombres, mientras que el desempleo femenino es aproximadamente un 37 \% mayor en la región. Por otro lado, las mujeres tienen el doble de probabilidades de trabajar a tiempo parcial (ILO/ECLAC 2019).

En gran medida, estas diferencias reflejan la persistencia de normas sociales sobre los roles de género y la división del trabajo doméstico no remunerado. Las encuestas sobre el uso del tiempo muestran que las mujeres siguen siendo responsables por aproximadamente el $75 \%$ del trabajo doméstico (incluido el cuidado de niños y miembros de la familia de edad avanzada), lo que limita la capacidad de obtener un empleo de tiempo completo (ILO/ECLAC 2019).

Existen diversos mecanismos a través de los cuales estas disparidades pueden dar como resultado diferentes oportunidades para el uso de Internet y el desarrollo de habilidades TIC, así como también distintos patrones de uso de Internet. En primer lugar, considérese el hecho de que menos de la mitad de todos los hogares en la región se suscriben a un servicio residencial de banda ancha (ITU 2019). Esto implica que para muchos el trabajo es el lugar elegido para conectarse a Internet y desarrollar habilidades TIC, muchas de las cuales no se pueden desarrollar mediante dispositivos móviles. El hecho de que muchos empleadores limiten el rango de aplicaciones disponibles para los empleados no disminuye la importancia del acceso a Internet en el lugar de trabajo. Tal como sucede con otras tecnologías de propósito general, la experiencia y la exposición continua (learning by doing) son fundamentales para desarrollar habilidades digitales en el trabajo, que son luego fácilmente transferibles al uso en otros contextos (Van Laar et al. 2017).

En segundo lugar, es probable que los empleadores inviertan en capacitación TIC para los trabajadores. Si bien estas inversiones en capital humano pueden estar limitadas a las tareas requeridas y los sistemas de información utilizados en una organización en particular, como se menciona anteriormente, muchas habilidades TIC son generalizables y fácilmente transferibles a otros contextos. En términos generales, la capacitación en el lugar de trabajo se asocia con una mayor familiaridad con los dispositivos digitales, con los sistemas informáticos, y con aplicaciones tales como el correo electrónico y los navegadores web. Es importante tener en cuenta que posiblemente esas inversiones en capital humano sean mayores en los sectores más intensivos en TIC y estén sesgadas hacia los empleados a tiempo completo, punto que retomaremos a continuación.

Por último, la participación laboral aumenta el costo de oportunidad del tiempo libre, 
creando así incentivos para que las personas accedan a Internet para minimizar el tiempo dedicado a tareas cotidianas tales como ir de compras o realizar operaciones bancarias. Dicho de otra manera, aquellos que están desempleados o subempleados tienen menores incentivos para encontrar alternativas en línea a las tareas cotidianas, y esto en última instancia puede resultar en niveles más bajos de uso de Internet.

Es evidente que cada uno de estos posibles vínculos entre el empleo y el uso de Internet afectará a hombres y mujeres de manera diferente. En términos generales, las diferencias de género en la participación en la fuerza laboral y el desempleo tienden a crear menos oportunidades para que las mujeres accedan a Internet en el lugar de trabajo. La Tabla 1 muestra que las diferencias de género en la participación laboral son amplias para los países en estudio (entre 17 p.p. en Perú y 34.6 p.p. en México). Dado el bajo nivel de desempleo en América Latina, las diferencias en las tasas de desempleo son algo menos relevantes pero aún significativas, particularmente en Ecuador, donde las mujeres tienen casi el doble de probabilidades de estar desempleadas.

Tabla 1. Género y empleo en países seleccionados de América Latina (2017)

\begin{tabular}{|c|c|c|c|c|c|c|c|c|}
\hline & \multicolumn{2}{|c|}{$\begin{array}{l}\text { Participación en } \\
\text { la fuerza laboral }\end{array}$} & \multicolumn{2}{|c|}{ Tasa de empleo } & \multicolumn{2}{|c|}{$\begin{array}{l}\text { Tasa de } \\
\text { desempleo }\end{array}$} & \multicolumn{2}{|c|}{ Horas semanales } \\
\hline & Hombre & Mujer & Hombre & Mujer & Hombre & Mujer & Hombre & Mujer \\
\hline Ecuador & 80,6 & 55,6 & 77,7 & 52,2 & 3,6 & 6,0 & 40,6 & 33,6 \\
\hline México & 77,6 & 43,0 & 75,0 & 41,4 & 3,3 & 3,6 & 49,7 & 38,9 \\
\hline Perú & 81,0 & 64,0 & 77,8 & 61,1 & 3,8 & 4,4 & 44,8 & 37,0 \\
\hline
\end{tabular}

Fuente: ILO y SEDLAC.

Menor participación en la fuerza laboral y mayor desempleo significan también que las mujeres tienen menos probabilidades de recibir capacitación en TIC por parte de sus empleadores. A su vez, es de conocimiento general que los empleadores están menos dispuestos a realizar inversiones de capital humano en trabajadores a tiempo parcial (Arulampalam y Booth 1998, Sobaih et al. 2011) que, como se muestra en la Tabla 1, afecta proporcionalmente más a las mujeres. Por otro lado, otros estudios sugieren que los empleadores discriminan a las mujeres en los programas de capacitación laboral (Altonji y Spletzer 1991, Green 1991, Barron et al. 1993, Evertsson 2004). En conjunto, estos hechos estilizados ayudan a explicar los resultados, que apuntan a niveles más altos de habilidades TIC entre los hombres en los tres países incluidos en este estudio (OECD 2016). ${ }^{[1]}$ 
Resulta interesante que la literatura sobre la brecha digital de género presta relativamente poca atención a las diferencias en los patrones de empleo entre hombres y mujeres. Esto es sorprendente dado que los estudios previos han encontrado consistentemente que estar ocupado está asociado con un mayor uso de Internet y con mayores niveles de alfabetización digital (Grazzi y Vergara 2014, Rashid 2016, Campos et al. 2017). Entre los pocos estudios que abordan esta temática se destaca Ono y Zavodny (2005), que analiza las diferencias de género en el empleo y en las habilidades TIC en Estados Unidos y Japón. Los autores encuentran una brecha digital de género significativamente mayor en Japón, que atribuyen al empleo desproporcionado de mujeres en trabajos no estándar a tiempo parcial donde la exposición a las TIC y la alfabetización digital son limitadas. De modo similar, Helsper (2010) encuentra que en el Reino Unido la situación laboral está asociada con diferentes actividades en línea para hombres y mujeres, pero que estas diferencias tienden a desaparecer a medida que los trabajadores alcanzan la edad de retiro del mercado laboral. Utilizando microdatos de seis países del África subsahariana, Alozie y Akpan-Obong (2017) encuentran que el empleo afecta positivamente la adopción de Internet entre los hombres, pero no así entre las mujeres.

Algunos pocos estudios examinan este tema en el contexto latinoamericano. Utilizando microdatos de encuestas para 12 países latinoamericanos, Hilbert (2011) encuentra que, al controlar por empleo, las diferencias de género en el uso de TIC tienden a desaparecer. Un hallazgo relacionado es que las mujeres informan mayor uso de Internet en el trabajo que los hombres, lo que sugiere que la contribución del empleo es mayor para las mujeres que para los hombres. Barrantes et al. (2019) utilizan datos de encuestas de 5 países (Argentina, Colombia, Guatemala, Perú y Paraguay) para examinar cómo diferentes factores contribuyen a la brecha digital de género, medidos a través de un índice que incluye el acceso a TIC, la experiencia y los tipos de uso. Los resultados sugieren que la situación laboral es, después del logro educativo, el segundo factor de mayor contribución a la brecha digital de género. Curiosamente, la parte no explicada de la brecha (es decir, no explicada por las diferencias en las características entre hombres y mujeres) es mayor en el centro de la distribución del índice. Esto sugiere la necesidad de considerar cómo los determinantes de la brecha digital de género varían según las características de grupo, un punto al que volveremos más adelante.

\section{Datos y metodología}

\subsection{Fuente de datos y muestra}

Las encuestas de donde provienen los datos utilizados en este estudio fueron administradas por las oficinas nacionales de estadística de los respectivos países. Son encuestas de hogares representativas a nivel nacional basadas en muestreo estratificado probabilístico para conglomerados urbanos y rurales. Cada oficina nacional de estadística proporciona los ponderadores de muestra individuales y de hogares que se ajustan a las tasas de no 
respuesta. Estos ponderadores se incluyen en los modelos de estimación presentados a continuación.

Existen numerosas ventajas en el uso de estas encuestas, en contraste con las encuestas adhoc de muestras más pequeñas. La primera ventaja es la calidad de los datos, ya que estas encuestas se basan en métodos rigurosos de muestreo y recopilación de datos. La segunda ventaja es el tamaño de la muestra, que varía de 29.605 casos válidos en Perú a 108.615 en México. Estas muestras permiten particiones de datos y el uso de estrategias empíricas inviables con muestras más pequeñas. Además, la recopilación de datos a través de entrevistas personales es crítica para los propósitos de este estudio, ya que los métodos de recolección que se basan en tecnologías de la información (tales como paneles en línea o muestreo telefónico aleatorio) pueden producir estimaciones sesgadas del uso de TIC (Bethlehem 2010).

Sin embargo, existen también varias limitaciones asociadas a estas encuestas. En primer lugar, existen variaciones significativas en los métodos de muestreo, en los tamaños de muestra y en los cuestionarios administrados en cada país. Debido a estas diferencias, este estudio analiza cada país por separado, y las comparaciones entre países deben interpretarse como indicativas de patrones comunes más que como estimaciones precisas de las diferencias. En la Tabla 2 se presentan las características de las encuestas en cada país. Dado el interés en cómo el empleo afecta el uso de Internet, el análisis restringe la muestra a la población adulta (mayor a 18 años).

Tabla 2. Encuestas y características de la muestra

\begin{tabular}{|c|c|c|c|c|}
\hline País & Encuesta & Fuente & $\begin{array}{l}\text { Tamaño } \\
\text { de la } \\
\text { muestra } \\
(18+)\end{array}$ & Año \\
\hline Ecuador & $\begin{array}{l}\text { Encuesta Nacional de } \\
\text { Empleo, Desempleo y } \\
\text { Subempleo (ENEMDU) }\end{array}$ & $\begin{array}{l}\text { Instituto Nacional de } \\
\text { Estadísticas y Censos } \\
\text { (INEC) }\end{array}$ & 69.172 & 2017 \\
\hline México & $\begin{array}{l}\text { Encuesta Nacional sobre } \\
\text { Disponibilidad y Uso de } \\
\text { Tecnologías de la } \\
\text { Información en los Hogares } \\
\text { (ENDUTIH) }\end{array}$ & $\begin{array}{l}\text { Instituto Nacional de } \\
\text { Estadística y Geografía } \\
\text { (INEGI) }\end{array}$ & 108.615 & 2018 \\
\hline Perú & $\begin{array}{l}\text { Encuesta Residencial de } \\
\text { Servicios de } \\
\text { Telecomunicaciones } \\
\text { (ERESTEL) }\end{array}$ & $\begin{array}{l}\text { Organismo Supervisor de } \\
\text { Inversión Privada en } \\
\text { Telecomunicaciones } \\
\text { (OSIPTEL) }\end{array}$ & 29.605 & 2018 \\
\hline
\end{tabular}


Fuente: elaboración propia.

Un segundo inconveniente se relaciona con los cuestionarios, que contienen generalmente un número relativamente pequeño de preguntas relacionadas con el acceso a Internet y con los tipos de uso. Esto limita la capacidad de analizar la adopción de Internet más allá de algunas características básicas, tal como el uso (vs. no uso) en un periodo de tiempo determinado, las horas de uso y el lugar más frecuente de uso. Además, existen diferencias en cómo se miden estas variables en las encuestas de cada país. Por ejemplo, en Ecuador se pregunta a los encuestados sobre el uso de Internet en los últimos 12 meses, mientras que en México la misma pregunta se refiere al uso en los últimos 3 meses. Además, en México y Perú los encuestados pueden seleccionar tantos lugares de uso de Internet como sea necesario, mientras que en Perú la selección de lugar de uso está limitada a tres opciones. Debido a estas y a otras diferencias en los cuestionarios, presentamos como los resultados para cada país por separado, interpretando resultados similares como tendencias comunes y no como diferencias precisas.

\subsection{Variables y estadísticas descriptivas}

Nuestra principal variable de interés es el uso de Internet y, más específicamente, las diferencias en el uso de Internet entre hombres y mujeres en función de la situación laboral. Las variables de control son las identificadas en la literatura como los determinantes más importantes del uso de Internet, tales como el ingreso, la educación, la edad, la composición familiar, la ubicación urbana-rural, y el acceso a Internet en el hogar (Fairlie 2004, Flamm y Chaudhuri 2007, Hauge y Prieger 2010, Galperín y Arcidiacono 2018, Manlove y Whitacre, 2019).

En la Tabla 3 se presentan las estadísticas descriptivas de nuestra principal variable de interés. Como se observa, la brecha de género en el uso de Internet es de aproximadamente 3 p.p. en Ecuador y México, aumentando a aproximadamente 6 p.p. en Perú. Si bien estas diferencias parecen relativamente pequeñas en comparación con otros países en desarrollo, son diferencias estadísticamente significativas y grandes en números absolutos. Por ejemplo, en el caso de México, la brecha representa una diferencia de aproximadamente 1.4 millones de mujeres adultas. Hay también diferencias de género en el lugar de uso más frecuente de Internet. Mientras que los hombres mencionan más frecuentemente el uso en el lugar de trabajo, las mujeres mencionan mayor uso en el hogar.

Sin embargo, estos resultados ignoran las diferencias de empleo y la división de género del trabajo doméstico no remunerado que, como se señaló en la Sección 2, recae desproporcionadamente en las mujeres. A modo de ejemplo, si la muestra se restringe a aquellos que están ocupados (a tiempo parcial o completo), la brecha en el uso de Internet desaparece (en Perú y Ecuador) o se invierte a favor de las mujeres (en México). Esto sugiere que la situación laboral no solo es un determinante importante del uso de Internet, sino también que el efecto puede ser diferente para las mujeres respecto de los hombres. Volveremos sobre este punto en la sección de resultados principales. 
Tabla 3. Estadísticas descriptivas (en \%)

\begin{tabular}{|c|c|c|c|c|c|c|c|c|c|}
\hline & \multicolumn{3}{|l|}{ Ecuador } & \multicolumn{3}{|l|}{ México } & \multicolumn{3}{|l|}{ Perú } \\
\hline & Hombre & Mujer & brecha & Hombre & Mujer & brecha & Hombre & Mujer & brecha \\
\hline $\begin{array}{l}\text { Uso de } \\
\text { Internet }\end{array}$ & 57.1 & 54.2 & $2.9 * * *$ & 65.3 & 61.9 & $3.4^{* * *}$ & 63.7 & 57.8 & $5.9 * * *$ \\
\hline \multicolumn{10}{|l|}{ Lugar de uso } \\
\hline Hogar & 70.3 & 73.9 & $-3.6^{* * *}$ & 88.5 & 90.7 & $-2.2^{* * *}$ & 36.7 & 37.1 & -0.4 \\
\hline $\begin{array}{l}\text { Lugar de } \\
\text { trabajo }\end{array}$ & 33.3 & 25 & $8.3^{* * *}$ & 62.6 & 42.8 & $19.8^{* * *}$ & 4.3 & 3.4 & $0.9 * *$ \\
\hline $\begin{array}{l}\text { Institución } \\
\text { educativa }\end{array}$ & 10.4 & 10.5 & -0.1 & 15.7 & 15.2 & 0.5 & 0.8 & 0.9 & -0.1 \\
\hline $\begin{array}{l}\text { Uso de } \\
\text { Internet } \\
\text { para } \\
\text { ocupados }\end{array}$ & 57.1 & 57.4 & -0.3 & 67.7 & 74.7 & $\begin{array}{l}-7.0 \\
* * *\end{array}$ & 65.7 & 65.4 & 0.3 \\
\hline Observaciones & 33,617 & 36,631 & & 51,889 & 57,781 & & 14,762 & 15,958 & \\
\hline
\end{tabular}

Fuente: elaboración propia.

Existe también evidencia de que la brecha digital de género no es homogénea entre distintos grupos según los niveles de ingresos y educación. La Figura 1 (panel izquierdo) muestra el ratio entre hombres y mujeres que utilizan Internet para diferentes niveles de logro educativo en cada uno de los países. Como se muestra, el ratio es significativamente mayor entre los de menor logro educativo, y tiende progresivamente a uno (es decir a la paridad de género) a medida que aumenta el nivel educativo. Se observa una tendencia similar, aunque menos consistente, para distintos niveles de ingresos, donde el ratio es mayor en el primer quintil de ingresos y tiende a la paridad entre los de mayores ingresos. ${ }^{[2]}$

Figura 1. Ratio de uso de Internet hombres/mujeres, según educación e ingresos 

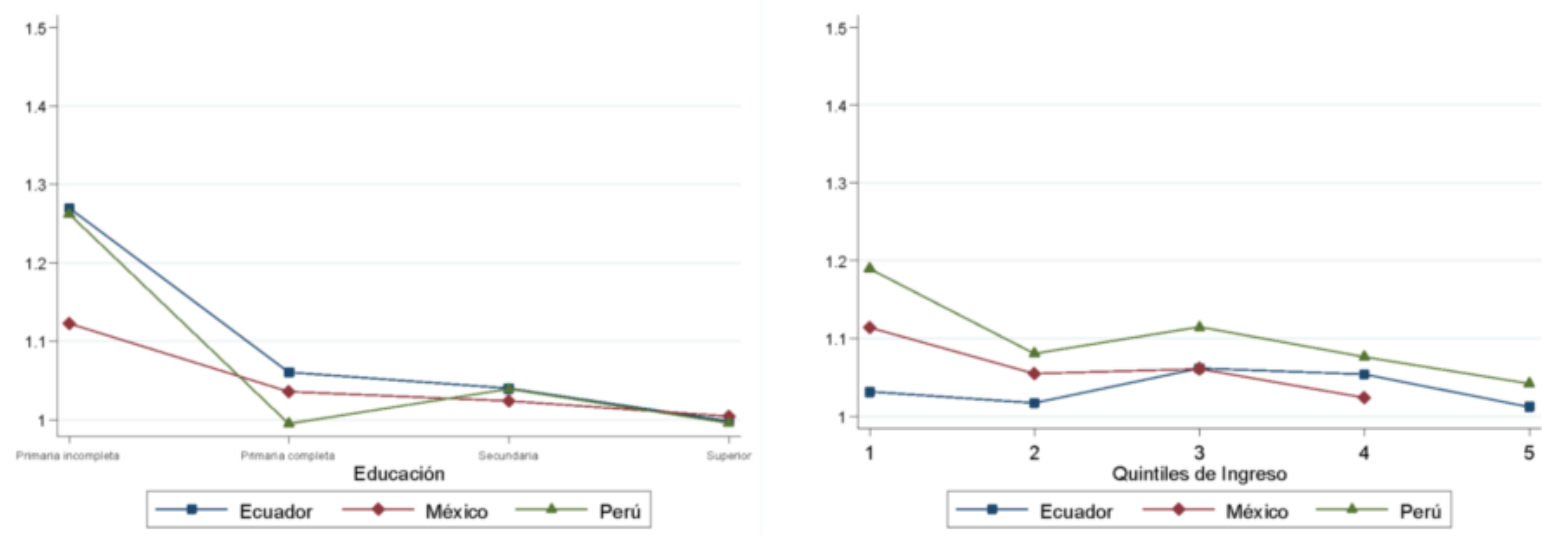

\subsection{Método}

El presente trabajo utiliza dos estrategias empíricas, cada una de las cuales ofrece un enfoque diferente para abordar nuestras preguntas de investigación. La primera se basa en un modelo logit que estima el efecto del empleo y otros factores demográficos en las probabilidades de uso de Internet. A partir de este modelo, calculamos la interacción entre género y empleo, con especial atención a las diferencias en el efecto del empleo en la adopción de Internet entre hombres y mujeres. Destacamos las diferencias en la escala aditiva, a menudo denominada contraste de interacción (IC).

La segunda estrategia utiliza una técnica de descomposición desarrollada por Fairlie (1999) que ayuda a identificar la contribución de cada factor a las diferencias observadas en el uso de Internet entre hombres y mujeres. Siguiendo el método de Blinder-Oaxaca (Blinder 1973), esta estrategia descompone la brecha digital de género en dos componentes, uno que captura las diferencias en las características observables o "dotaciones" entre hombres y mujeres (por ejemplo, diferencias en la participación laboral) y uno que captura las diferencias en los "coeficientes" o, en otras palabras, en el efecto de estas características en las probabilidades de uso de Internet, nuestro resultado de interés.

La técnica de descomposición original se desarrolló para modelos lineales y, como lo demuestra Fairlie (2005), puede conducir a estimaciones sesgadas cuando el resultado de interés es binario, como es nuestro caso. Como solución a este problema, Fairlie utiliza un algoritmo de simulación que permite la descomposición utilizando la ecuación no lineal original (probit o logit). En el contexto de este estudio, la brecha digital de género Yhombre- Ymujer puede descomponerse de la siguiente manera: 


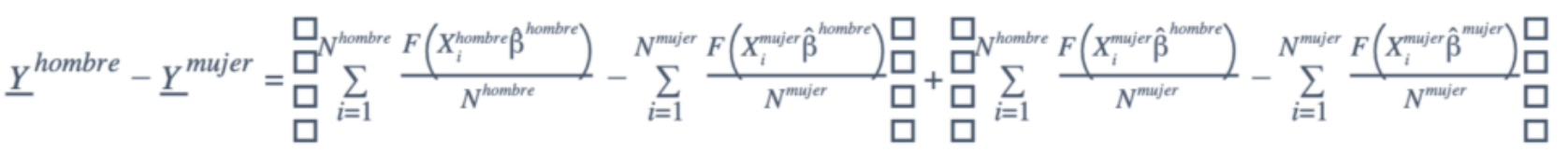

(1)

donde $\mathrm{Nj}$ es el tamaño de muestra para el género j, Xij es un vector fila de i variables independientes, y jes un vector columna de coeficientes. El primer término entre paréntesis representa la parte de la brecha digital de género que puede atribuirse a las diferencias de género en las características observables Xi, mientras que el segundo término representa la parte atribuible a las diferencias de género en los coeficientes junto con cualquier efecto atribuible a variables no observadas. Dada esta ambigüedad en la interpretación del segundo término, enfatizamos los resultados del primer término, que crea un contrafactual al calcular la diferencia entre la probabilidad predicha para los hombres usando sus propios coeficientes y características grupales, y la misma probabilidad usando las características

de las mujeres. Esto es similar a plantear la pregunta hipotética: “iy si los hombres tuvieran las características observables de las mujeres?". Una ecuación igualmente válida es (2), donde en el primer término los coeficientes de las mujeres se combinan con las características observables de los hombres:

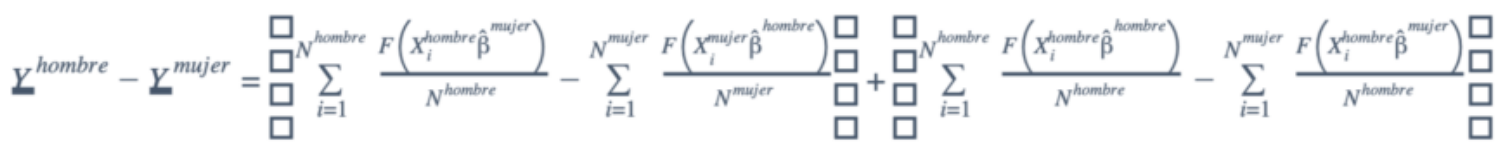

(2)

Los resultados obtenidos de (1) y (2) pueden diferir porque usan diferentes grupos de referencia, lo que se conoce en la literatura de descomposición como el problema del índice (Oaxaca 1973). A priori, ambos escenarios hipotéticos son igualmente informativos para comprender la brecha de género digital. Por lo tanto, presentamos ambos conjuntos de resultados que, como se discute a continuación, son cualitativamente similares, lo que facilita la interpretación. También es importante tener en cuenta que debido a que el segundo término en las ecuaciones captura la variación atribuible a variables no observadas, la suma de las contribuciones de las variables individuales no alcanza el $100 \%$ $y$, en algunos casos particulares, puede incluso superar este umbral.

A partir de las ecuaciones (1) y (2) es también posible estimar la contribución de cada factor a la brecha digital de género. Esto se realiza calculando el cambio en la probabilidad predicha promedio al reemplazar los valores de una sola variable para un grupo con los valores para el otro grupo mientras se mantienen constantes los valores de las otras 
variables. Siguiendo las principales preguntas de investigación, el énfasis del análisis está en la contribución de las diferencias de género en el empleo en relación con la contribución de otros predictores.

\section{Resultados}

Comenzamos presentando los resultados de la primera estrategia de estimación. El modelo logit (Tabla 4) estima las probabilidades de uso de Internet condicionadas en características demográficas observables. Como se observa, las variables predictoras tienen el efecto esperado: la edad está inversamente relacionada con la probabilidad de uso de Internet, mientras que la educación, el ingreso, la presencia de niños en el hogar, la ubicación urbana y la suscripción a banda ancha residencial aumentan la probabilidad de uso de Internet (la excepción es el número de miembros del hogar que, contrariamente a lo esperado, reduce las probabilidades de uso).

Volviendo a nuestras variables de interés, en primer lugar observamos que, ceteris paribus, las mujeres tienen menor probabilidad de reportar uso de Internet en Ecuador y Perú, y la magnitud del efecto es relativamente grande. Interpretando los resultados en razón de probabilidades (odds ratios), se observa que las probabilidades de uso de Internet entre mujeres son entre 16 \% (en Ecuador) y 19 \% (en Perú) menores respecto de los hombres. Curiosamente, no se detecta ningún efecto de género en el caso de México. Nuestra principal variable de interés es la situación laboral, que demuestra ser un fuerte predictor del uso de Internet en los tres países. En términos de razón de probabilidades, estar ocupado incrementa la probabilidad de uso de Internet en un rango que varía del $22 \%$ (en Ecuador) al 69 \% (en México).

Tabla 4. Probabilidad de uso de Internet (en coeficientes logit) 


\begin{tabular}{|c|c|c|c|}
\hline & Ecuador & México & Perú \\
\hline \multirow[t]{2}{*}{ Mujer (=1) } & $-0,178$ & 0,018 & $-0,208$ \\
\hline & $(0,039)^{* * *}$ & $(0,041)$ & $(0,052)^{* * *}$ \\
\hline \multirow[t]{2}{*}{ Ocupado (=1) } & 0,199 & 0,526 & 0,486 \\
\hline & $(0,046)^{* * *}$ & $(0,045)^{* * *}$ & $(0,056)^{* * *}$ \\
\hline \multirow[t]{2}{*}{ Edad } & $-0,076$ & $-0,096$ & $-0,087$ \\
\hline & $(0,008)^{* * *}$ & $(0,007)^{* * *}$ & $(0,009)^{* * *}$ \\
\hline \multirow[t]{2}{*}{$($ Edad $) 2$} & $-0,000$ & 0,000 & 0,000 \\
\hline & $(0,000)^{* * *}$ & $(0,000)$ & $(0,000)^{* *}$ \\
\hline \multirow[t]{2}{*}{ Educación secundaria incompleta } & 1,082 & 1,061 & 0,474 \\
\hline & $(0,050)^{* * *}$ & $(0,043)^{* * *}$ & $(0,091)^{* * *}$ \\
\hline \multirow[t]{2}{*}{ Educación secundaria completa } & 1,476 & 1,825 & 1,332 \\
\hline & $(0,046)^{* * *}$ & $(0,060)^{* * *}$ & $(0,064)^{* * *}$ \\
\hline \multirow[t]{2}{*}{ Educación superior } & 2,717 & 2,841 & 2,397 \\
\hline & $(0,067)^{* * *}$ & $(0,074)^{* * *}$ & $(0,076)^{* * *}$ \\
\hline \multirow[t]{2}{*}{ Ingreso del hogar per capita (log) } & 0,555 & & 0,400 \\
\hline & $(0,026)^{* * *}$ & & $(0,034)^{* * *}$ \\
\hline \multirow[t]{2}{*}{ Nivel Socioeconómico $=2$} & & 0,709 & \\
\hline & & $(0,055)^{* * *}$ & \\
\hline \multirow{2}{*}{ Nivel Socioeconómico $=3$} & & 1,127 & \\
\hline & & $(0,072)^{* * *}$ & \\
\hline \multirow[t]{2}{*}{ Nivel Socioeconómico $=4$} & & 1,366 & \\
\hline & & $(0,113)^{* * *}$ & \\
\hline \multirow[t]{2}{*}{ Hogar urbano $(=1)$} & 0,736 & 0,396 & 0,817 \\
\hline & $(0,040)^{* * *}$ & $(0,049)^{* * *}$ & $(0,081)^{* * *}$ \\
\hline \multirow[t]{2}{*}{ Presencia de niños $(=1)$} & 0,188 & 0,079 & 0,140 \\
\hline & $(0,046)^{* * *}$ & $(0,044)^{*}$ & $(0,058)^{* *}$ \\
\hline \multirow[t]{2}{*}{ Miembros en el hogar } & $-0,042$ & $-0,112$ & $-0,136$ \\
\hline & $(0,011)^{* * *}$ & $(0,014)^{* * *}$ & $(0,017)^{* * *}$ \\
\hline \multirow[t]{2}{*}{ Acceso a Internet residencial $(=1)$} & 1,688 & 1,960 & 4,070 \\
\hline & $(0,044)^{* * *}$ & $(0,042)^{* * *}$ & $(0,085)^{* * *}$ \\
\hline \multirow[t]{2}{*}{ Constante } & $-1,242$ & 1,578 & $-3,823$ \\
\hline & $(0,202)^{* * *}$ & $(0,164)^{* * *}$ & $(0,293)^{* * *}$ \\
\hline Observaciones & 69.172 & 108.615 & 29.605 \\
\hline
\end{tabular}


Notas:

1. Errores estándar robustos entre paréntesis.

2. $* * * \mathrm{p}<0.01, * * \mathrm{p}<0.05, * \mathrm{p}<0.1$.

3. La escuela primaria incompleta es la categoría de referencia para logro educativo. Fuente: elaboración propia.

De modo general, estos resultados validan los hallazgos de estudios previos que apuntan al género y al empleo como predictores de la adopción de Internet. Sin embargo, nuestra hipótesis de trabajo es que, debido a las diferencias en los patrones de empleo, estar ocupado afecta el uso de Internet de manera diferente para hombres y mujeres. Para examinar esta pregunta, calculamos la diferencia entre las probabilidades predichas en los siguientes cuatro grupos: hombre ocupado (HO), hombre no ocupado (HN), mujer ocupada (MO) y mujer no ocupada (MN). Esto permite obtener la interacción aditiva o el contraste de interacción (IC), que se puede expresar de la siguiente manera:

$I C=[\operatorname{Pr}(H O)-\operatorname{Pr}(H N)]-[\operatorname{Pr}(M O)-\operatorname{Pr}(M N)]$

La Tabla 5 presenta las diferencias en las probabilidades predichas del uso de Internet para hombres y mujeres según situación laboral, así como el contraste de interacción (IC) resultante. Como se observa, estar ocupado tiene un mayor efecto aditivo en la probabilidad de uso de Internet para las mujeres que para los hombres. En el caso de Ecuador, la situación laboral tiene un efecto nulo sobre la probabilidad de uso de Internet para los hombres, pero, en el caso de las mujeres, la probabilidad aumenta en casi 8 p.p.. En México y Perú encontramos que el estar ocupado aumenta la probabilidad de uso para ambos sexos, pero, como se muestra, el aumento (en p.p.) es casi el doble para las mujeres. En general, estos hallazgos validan la hipótesis de que, si bien la situación laboral es un contribuyente significativo a la adopción de Internet para ambos sexos, el efecto es significativamente mayor para las mujeres.

Tabla 5. Probabilidad de uso de Internet, según género y situación de empleo 


\begin{tabular}{|c||c||c||l|}
\hline & $\begin{array}{l}1 . \mathrm{Hombre} \\
{[\operatorname{Pr}(\mathrm{HO})-\operatorname{Pr}(\mathrm{HN})]}\end{array}$ & $\begin{array}{l}\text { 1. Mujer } \\
{[\operatorname{Pr}(\mathrm{MO})-\operatorname{Pr}(\mathrm{MN})]}\end{array}$ & $\begin{array}{l}\text { Contraste de Interacción } \\
(\mathrm{A}-\mathrm{B})\end{array}$ \\
\hline \hline Ecuador & 0,000 & $0,077^{* * *}$ & $-0,076^{* * *}$ \\
\hline \hline & $(0,007)$ & $(0,006)$ & $(0,009)$ \\
\hline \hline México & $0,140^{* * *}$ & $0,255^{* * *}$ & \\
\hline \hline & $(0,007)$ & $(0,006)$ & $-0,115^{* * *}$ \\
\hline \hline & & & $(0,009)$ \\
\hline \hline Perú & $0,093^{* * *}$ & $0,142^{* * *}$ & $-0,049 * * *$ \\
\hline \hline & $(0,008)$ & $(0,006)$ & $(0,011)$ \\
\hline
\end{tabular}

Notas:

1. Errores estándar robustos entre paréntesis.

2. $* * * \mathrm{p}<0.01, * * \mathrm{p}<0.05, * \mathrm{p}<0.1$.

Fuente: elaboración propia.

La Figura 2 ofrece una visualización de estos resultados. En cada panel, la línea roja representa el cambio en las probabilidades predichas de uso de Internet para las mujeres asociadas con el empleo, mientras que la línea azul representa el cambio en los mismos valores para los hombres. Como se observa, si las mujeres trabajaran a la misma tasa que los hombres, la brecha digital de género desaparecería en Perú y en Ecuador. En el caso de México, la brecha se revertiría en favor de las mujeres.

Figura 2. Probabilidad predicha de uso de Internet, según género y situación laboral 

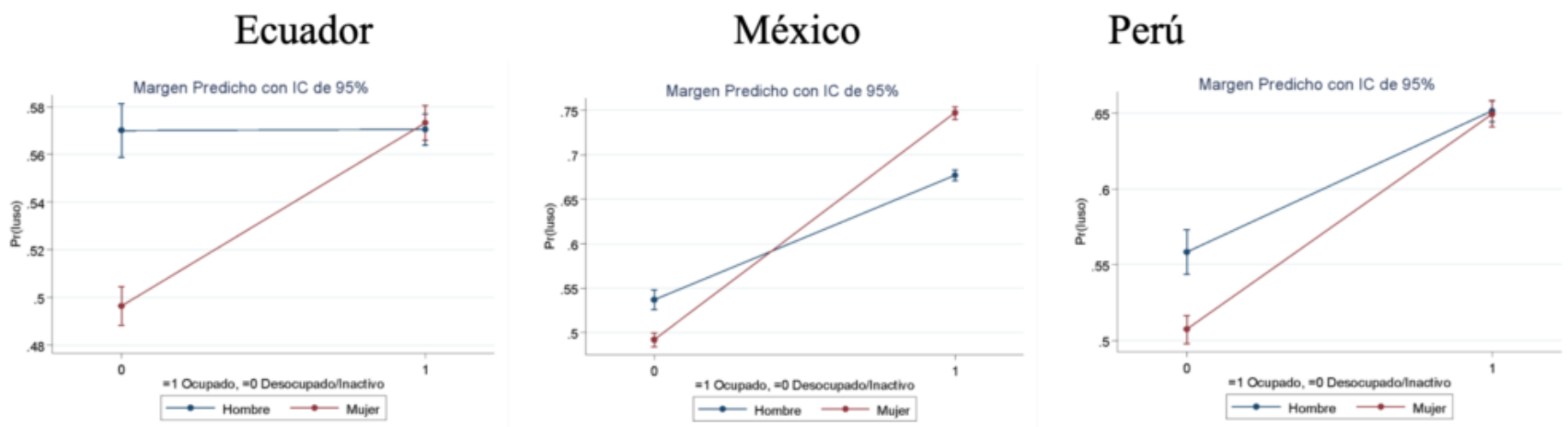

Hay también evidencia de que el efecto positivo del empleo femenino en las probabilidades de uso de Internet varía significativamente según los estratos socioeconómicos. Estas diferencias se representan en la Figura 3, la cual representa el cambio esperado en las probabilidades de adopción de Internet por efecto del empleo ${ }^{[3]}$ para diferentes quintiles de ingresos o niveles socioeconómicos en el caso de México. Los resultados muestran que el efecto del empleo femenino en el uso de Internet aumenta con los ingresos, con la única excepción de México, donde cae para el nivel socioeconómico más alto. Es interesante notar que en dos de los tres países el efecto es nulo (Perú) o incluso negativo (Ecuador) en los quintiles de ingresos más bajos, lo que sugiere que el empleo femenino en estos estratos se concentra en ocupaciones que ofrecen pocas o ninguna oportunidad de acceso y capacitación en TIC, tal como en el sector de servicios domésticos.

Figura 3. Cambio en las probabilidades de uso de Internet para mujeres, según ingreso o estrato socioeconómicos
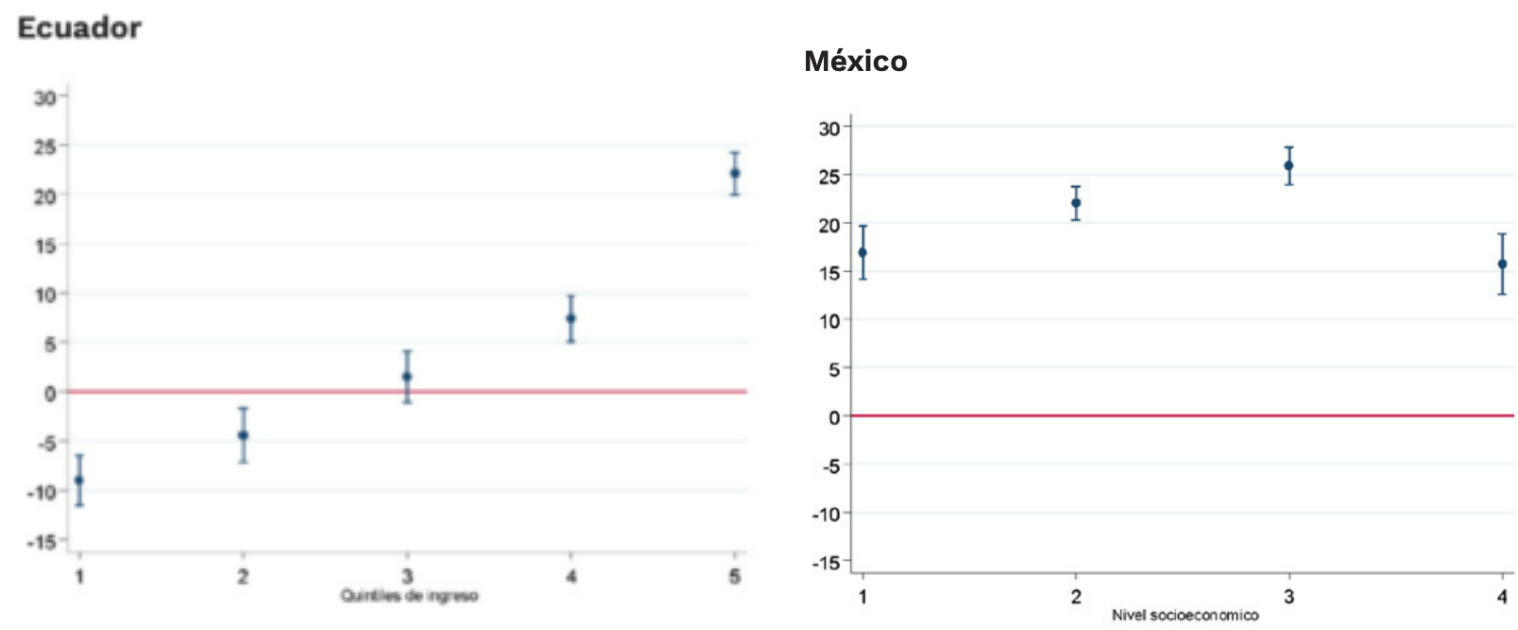


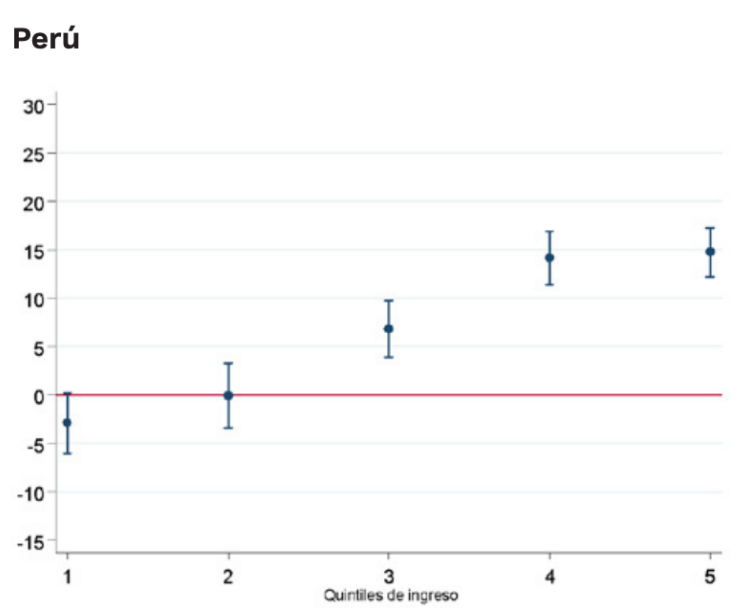

La segunda estrategia empírica descompone la brecha digital de género en sus factores contribuyentes. La Tabla 6 presenta dos conjuntos de resultados para cada variable predictiva. El primer conjunto utiliza los coeficientes para hombres como grupo de referencia (Ecuación 1) y el segundo utiliza los coeficientes para mujeres como grupo de referencia (Ecuación 2). Como se muestra, si bien existen algunas diferencias en las magnitudes, ambos conjuntos de resultados arrojan una conclusión similar: las diferencias en la participación laboral entre hombres y mujeres son el factor que más contribuye a la brecha digital de género. Además, la contribución relativa de las diferencias de género en el empleo es significativamente mayor que la contribución de otras variables, excepto en un modelo para Perú donde las diferencias en condición laboral son el segundo factor de mayor peso, marginalmente por detrás del logro educativo.

Incluso cuando se utilizan las estimaciones de límite inferior para cada país, los resultados sugieren que las diferencias en empleo son responsables de aproximadamente una cuarta parte de la brecha en el uso de Internet entre hombres y mujeres. La única otra variable que tiene una contribución de tamaño similar es el logro educativo, que en México y Perú es responsable de aproximadamente una cuarta parte de la brecha digital de género. En el caso de Ecuador, las diferencias de género en los ingresos parecen contribuir más que las diferencias en el nivel educativo. Sin embargo, la contribución es significativamente menor que la del empleo. En general, los resultados de la descomposición validan la hipótesis respecto de que las brechas de género en la participación en la fuerza laboral, las tasas de desempleo y el empleo a tiempo parcial conducen a diferentes oportunidades de acceso a Internet y capacitación digital entre hombres y mujeres en la región.

La porción de la brecha que puede explicarse a través de las diferencias de género en las características observables difiere entre los países estudiados. En México y Perú, las variables incluidas en el modelo explican gran parte (aproximadamente tres cuartos) de la brecha de género digital, pero en el caso de Ecuador, la porción de la brecha explicada por las características observables es de solo un tercio. A pesar del problema en la interpretación del segundo término de la descomposición (discutido anteriormente), esto sugiere que las diferencias en los coeficientes o, en otras palabras, en el efecto del empleo 
en hombres y mujeres, son más relevantes que las diferencias en la situación laboral per se. Esto es consistente con los resultados del contraste de interacción, que sugieren que el uso de Internet en Ecuador es similar para los hombres independientemente del estado laboral, mientras que para el caso de las mujeres el empleo aumenta las probabilidades de uso de Internet en aproximadamente 8 p.p., como se muestra en la Tabla 6.

Tabla 6. Descomposición de diferencias en uso de Internet 


\begin{tabular}{|c|c|c|c|c|c|c|}
\hline \multirow[b]{2}{*}{ Al usar coeficientes de } & \multicolumn{2}{|l|}{ Ecuador } & \multicolumn{2}{|l|}{ México } & \multicolumn{2}{|l|}{ Perú } \\
\hline & Hombre & Mujer & Hombre & Mujer & Hombre & Mujer \\
\hline Ocupado & $0,006^{*}$ & $0,007^{* * *}$ & $0,010^{* * *}$ & $0,025^{* * *}$ & $0,027^{* * *}$ & $0,014^{* * *}$ \\
\hline SE & 0,003 & 0,002 & 0,003 & 0,002 & 0,004 & 0,003 \\
\hline Contribución relativa & $19,2 \%$ & $22,2 \%$ & $31,5 \%$ & $74,9 \%$ & $45,7 \%$ & $24,4 \%$ \\
\hline Presencia de niños & $-0,002 * * *$ & 0,000 & 0,000 & 0,000 & 0,000 & 0,000 \\
\hline SE & 0,000 & 0,000 & 0,000 & 0,000 & 0,000 & 0,000 \\
\hline Contribución relativa & $-6,0 \%$ & $-0,3 \%$ & $-1,1 \%$ & $-0,7 \%$ & $-0,2 \%$ & $-0,7 \%$ \\
\hline Miembros en el hogar & 0,000 & 0,000 & 0,000 & 0,000 & $-0,001^{* * *}$ & 0,000 \\
\hline SE & 0,000 & 0,000 & 0,000 & 0,000 & 0,000 & 0,000 \\
\hline Contribución relativa & $0,9 \%$ & $0,8 \%$ & $0,5 \%$ & $1,0 \%$ & $-1,2 \%$ & $-0,6 \%$ \\
\hline Edad & $0,002^{* * *}$ & $0,002^{* * *}$ & $0,006^{* * *}$ & $0,006^{* * *}$ & $-0,005^{* * * *}$ & $-0,004^{* * *}$ \\
\hline SE & 0,001 & 0,001 & 0,001 & 0,001 & 0,001 & 0,001 \\
\hline Contribución relativa & $5,6 \%$ & $6,6 \%$ & $18,7 \%$ & $16,9 \%$ & $-8,0 \%$ & $-6,2 \%$ \\
\hline Hogar urbano & 0,000 & 0,000 & 0,000 & 0,000 & 0,000 & 0,000 \\
\hline $\mathrm{SE}$ & 0,000 & 0,000 & 0,000 & 0,000 & 0,000 & 0,000 \\
\hline Contribución relativa & $-0,6 \%$ & $-0,5 \%$ & $-0,1 \%$ & $-0,2 \%$ & $-0,2 \%$ & $-0,6 \%$ \\
\hline Educación & $0,001 * * *$ & $0,001^{* * *}$ & $0,009^{* * *}$ & $0,008^{* * *}$ & $0,018^{* * *}$ & 0,019 *** \\
\hline SE & 0,001 & 0,001 & 0,001 & 0,001 & 0,001 & 0,001 \\
\hline Contribución relativa & $4,7 \%$ & $4,7 \%$ & $26,2 \%$ & $24,7 \%$ & $29,9 \%$ & $32,5 \%$ \\
\hline Ingreso del hogar p.c. & $0,006^{* * *}$ & $0,005^{* * *}$ & $-0,001$ & $-0,001 *$ & $0,003^{* * *}$ & $0,003^{* * *}$ \\
\hline $\mathrm{SE}$ & 0,000 & 0,000 & 0,000 & 0,000 & 0,000 & 0,000 \\
\hline Contribución relativa & $18,9 \%$ & $15,6 \%$ & $-1,6 \%$ & $-1,9 \%$ & $4,3 \%$ & $4,8 \%$ \\
\hline Acceso residencial & $-0,003^{* * *}$ & $-0,003^{* * *}$ & 0,000 & 0,000 & $0,004^{* * *}$ & $0,004^{* * *}$ \\
\hline SE & 0,001 & 0,000 & 0,001 & 0,001 & 0,001 & 0,001 \\
\hline Contribución relativa & $-9,1 \%$ & $-11,1 \%$ & $-0,3 \%$ & $1,5 \%$ & $6,7 \%$ & $7,0 \%$ \\
\hline Contribución características (A) & 0,010 & 0,012 & 0,024 & 0,038 & 0,045 & 0,035 \\
\hline Brecha observada (B) & 0,031 & 0,031 & 0,033 & 0,033 & 0,059 & 0,059 \\
\hline Brecha explicada (A/B) & $33,3 \%$ & $38,1 \%$ & $73,6 \%$ & $115,9 \%$ & $77,5 \%$ & $60,5 \%$ \\
\hline \# de Observaciones & 69.172 & 69.172 & 108.615 & 108.615 & 29.605 & 29.605 \\
\hline
\end{tabular}


Nota:*** $\mathrm{p}<0.01, * * \mathrm{p}<0.05, * \mathrm{p}<0.1$

Fuente: elaboración propia.

\section{Discusión y conclusiones}

La brecha digital de género no emerge en un vacío. Más bien, la irrupción de nuevas tecnologías de comunicación como Internet ha estado determinada por fuerzas sociales que por mucho tiempo han favorecido a los hombres a través de una variedad de mecanismos institucionales y normativos. Estas fuerzas son particularmente relevantes en varios países emergentes donde la paridad de género todavía está rezagada, y para grupos sociales entre los cuales predominan expectativas y normas sociales tradicionales respecto a los roles de género.

América Latina presenta un caso interesante en este sentido. La región ha experimentado importantes cambios legales y culturales en los últimos 50 años que han reducido significativamente las brechas de género en educación, representación política y en varias otras áreas clave (Bando 2019). Como ejemplo, las jóvenes latinoamericanas (de 25 a 34 años) ahora tienen más educación que los hombres de dicho rango etario, y la mayoría de los países han promulgado leyes que prohíben la discriminación de género en la contratación laboral (Pagés y Piras 2010). Sin embargo, los mercados laborales de la región siguen estando fuertemente determinados por los estereotipos de género, así como por las barreras institucionales persistentes (por ejemplo, las diferencias en el permiso parental para hombres y mujeres), que funcionan en contra de las trabajadoras mujeres (actuales o potenciales).

Tal como fue mencionado en la introducción, diversos estudios han reconocido al empleo como un importante predictor del uso de Internet. Los trabajadores a menudo adquieren experiencia y capacitación en TIC en el lugar de trabajo y esas habilidades son fácilmente transferibles a otras dimensiones de uso de Internet. Además, tener acceso a Internet en el trabajo es aún más importante para aquellos cuyas opciones de conectividad se limitan a la banda ancha móvil, un servicio a menudo restringido por límites de datos, aplicaciones bonificadas y capacidades limitadas del dispositivo. Vale la pena recordar que la mayoría de las personas en América Latina acceden a Internet a través de una suscripción móvil prepaga, y menos de la mitad de los hogares de la región están conectados al servicio residencial de Internet (ITU 2019).

A pesar de estos hechos, la literatura sobre brechas digitales de género ha prestado sorprendentemente poca atención al nexo entre mercados laborales y uso de Internet. Este estudio contribuye a llenar este vacío al explorar cómo las diferencias de género en el empleo afectan la brecha digital de género en tres países latinoamericanos. Los hallazgos apuntan de manera concluyente al empleo como el factor contribuyente más relevante para la brecha de uso de Internet entre hombres y mujeres, por delante de otros predictores 
tradicionales como la edad, la educación y los ingresos. Según nuestras estimaciones, si las mujeres estuvieran empleadas a la misma tasa que los hombres, la brecha digital de género se reduciría al menos en una cuarta parte.

También encontramos evidencia de que la situación laboral tiene un mayor impacto en las probabilidades de estar en línea para las mujeres que para los hombres, posiblemente debido a las diferencias de género en las ocupaciones. Las mujeres están sobrerrepresentadas en sectores laborales como educación, salud, administración pública y otros sectores intensivos en TIC, mientras que están subrepresentadas en la construcción, el transporte y otras ocupaciones que han sido más lentas en adoptar nuevas tecnologías de la información (Gasparini et al. 2015).

Sin embargo, los resultados para Ecuador y Perú (y en menor medida para México) también sugieren que este efecto es heterogéneo entre los estratos socioeconómicos. En particular, sugieren que el efecto del empleo en el uso de Internet aumenta con el nivel socioeconómico, pero es nulo (Perú) o incluso negativo (Ecuador) entre las mujeres de bajos ingresos, quienes tienden a estar ocupadas de manera informal en empleos de baja productividad y baja intensidad tecnológica (ECLAC 2013). Estos resultados sugieren la necesidad de políticas específicas que rompan el ciclo por el cual las mujeres de bajos ingresos quedan excluidas del empleo formal debido a la baja alfabetización digital pero, al mismo tiempo, tienen menos oportunidades de adquirir dichas habilidades debido a la limitada participación laboral, o bien debido al empleo en ocupaciones con pocas oportunidades de acceso y capacitación en TIC.

Mirando hacia el futuro, también es necesario considerar cómo los cambios en la estructura del empleo como resultado de la digitalización y la inteligencia artificial pueden afectar la brecha digital de género en la región. Existe evidencia de que la nueva ola de automatización está afectando el empleo en ocupaciones de servicios de baja calificación con tareas cognitivas de rutina donde las mujeres están sobrerrepresentadas, tales como el comercio minorista y los servicios al cliente (Weller et al. 2019). Por otro lado, las mujeres tienden a trabajar en empresas más pequeñas y de menor productividad que están más alejadas de la frontera tecnológica. Otros estudios sugieren que las oportunidades de trabajo remoto en la gig economy promoverán el ingreso de las mujeres a la fuerza laboral debido a la flexibilidad en las horas de trabajo y las horas trabajadas (ILO/ECLAC, 2019). Sin embargo, la evidencia hasta ahora indica que la gran mayoría de los trabajadores remotos en América Latina son hombres (Berg 2018, Madariaga y Ernst 2019), lo que en parte refleja la brecha de género en la experiencia y la alfabetización digital explorada en este estudio.

Finalmente, si estos hallazgos son representativos de otros países de la región, o de países emergentes en general, sigue siendo una pregunta abierta. Los hallazgos para México y Perú sugieren que las características observables explican una gran parte (aproximadamente tres cuartos) de la brecha digital de género. Sin embargo, el caso de 
Ecuador sugiere que las diferencias entre países en la estructura del mercado laboral también pueden afectar la forma en que el empleo afecta el uso de Internet para hombres y mujeres. Por ejemplo, en países con mayor empleo en el sector de servicios, la recompensa a la participación de la mujer en la fuerza laboral probablemente sea mayor, mientras que lo contrario pueda ser cierto para los países con una mayor participación del empleo agrícola. Como mínimo, los casos presentados aquí proporcionan una línea de base contra la cual comparar los hallazgos de otros países y regiones.

\section{Referencias bibliográficas}

Alozie, Nicholas O. y Patience Akpan-Obong. 2017. The digital gender divide: Confronting obstacles to women's development in Africa. Development Policy Review, 35, № 2, pp. 137-160.

Altonji, Joseph G. y James R. Spletzer. 1991. Worker characteristics, job characteristics, and the receipt of on-the-job training. ILR Review, 45, № 1, pp. 58-79.

Arulampalam, Wiji y Alison L. Booth. 1998. Training and labour market flexibility: is there a trade-off?. British Journal of Industrial Relations, 36, № 4, pp. 521-536.

Bando, Rosangela. 2019. Evidence-based gender equality policy and pay in Latin America and the Caribbean: Progress and challenges. Latin American Economic Review, 28(10).

Barrantes, Roxana, Aileen Aguero García y Paulo Matos. 2019. Decomposing the ICT Use Gender Gap for Five Latin American Countries. Presentado en 13th CPR LATAM conference, Córdoba, Argentina.

Barron, John M., Dan A. Black y Mark A. Loewenstein. 1993. Gender differences in training, capital, and wages. Journal of human Resources, pp. 343-364.

Bethlehem, Jelke. 2010. Selection Bias in Web Surveys. International Statistical Review, 78(2), pp. 161-188.

Berg, Janine (2018). Digital labor platforms and the future of work: Towards decent work in the online world. Ginebra: ILO.

Berg, Janine, Marianne Furrer, Ellie Harmon, Uma Rani y Michael Six Silberman. 2018. Digital labour platforms and the future of work: Towards decent work in the online world. Ginebra: International Labour Office.

Bhandari, Aarushi. 2019. Gender inequality in mobile technology access: the role of economic and social development. Information, Communication and Society 22, № 5.

Blinder, Alan S. 1973. Wage Discrimination: Reduced form and structural estimates. Journal of Human Resources, 8, pp. 436-455. 
Campos, Raquel, María Arrazola y José de Hevia. 2017. Economic Crisis and Benefits of the Internet: Differentiated Internet Usage by Employment Status. Economics of Innovation and New Technology 26, № 3, pp. 269-294.

ECLAC. 2013. Women in the digital economy: breaking through the equality threshold.

Evertsson, Marie. 2004. Formal on-the-job training: A gender-typed experience and wagerelated advantage?. European Sociological Review, 20, № 1, pp. 79-94.

Fairlie, Robert W. 1999. The Absence of the African-American Owned Business: An Analysis of the Dynamics of Self-Employment. Journal of Labor Economics, 17, № 1, pp. 80-108.

Fairlie, Robert W. 2004. Race and the digital divide. Contributions to Economic Analysis and Policy, 3, № 1.

Fairlie, Robert W. 2005. An Extension of the Blinder-Oaxaca Decomposition Technique to Logit and Probit Models. Journal of Economic and Social Measurement, 30, № 4, pp. 305-316.

Flamm, Kenneth y Anindya Chaudhuri. 2007. An analysis of the determinants of broadband access. Telecommunications Policy, 31, № 6-7, pp. 312-326.

Galperín, Hernán y Malena Arcidiacono. 2018. Learning from or leaning on? The impact of children on Internet use by adults. New Media \& Society, 21, № 2, pp. 483-506.

Gasparini, Leonardo, Mariana Marchionni, Nicolás Badaracco y Joaquín Serrano. 2015. Female Labor Force participation in Latin America: evidence of deceleration. Documentos de Trabajo del CEDLAS.

Gray, Tricia J., Jason Gainous y Kevin M. Wagner. 2017. Gender and the digital divide in Latin America. Social Science Quarterly, 98, № 1, pp. 326-340.

Grazzi, Matteo y Sebastian Vergara. 2014. Internet in Latin America: who uses it?... and for what?. Economics of Innovation and New Technology, 23, № 4, pp. 327-352.

Green, Francis. 1991. Sex discrimination in job-related training. British Journal of Industrial Relations 29, № 2, pp. 295-304.

GSMA, Women, Connected. 2019. The mobile gender gap report 2018.

Hauge, Janice A. y James E. Prieger. 2010. Demand-side programs to stimulate adoption of broadband: What works?. Review of Network Economics 9, № 3.

Helsper, Ellen Johanna. 2010. Gendered internet use across generations and life stages. Communication research 37, № 3, pp. 352-374.

Hilbert, Martin. 2011. Digital gender divide or technologically empowered women in developing countries? A typical case of lies, damned lies, and statistics. Women's Studies 
International Forum, vol. 34, № 6, pp. 479-489. Pérgamo.

ILO/ECLAC. 2019. Employment Situation in Latin America and the Caribbean: Evolution of and prospects for women's labor participation in Latin America.

ITU. 2019. Facts and Figures. Ginebra.

Madariaga, Javier, César Buenadicha, Erika Molina y Christoph Ernst. 2019. Economía de plataformas y empleo: ¿Cómo es trabajar para una app en Argentina?, Vol. 718. InterAmerican Development Bank.

Manlove, Jacob y Brian Whitacre. 2019. Understanding the trend to mobile-only internet connections: A decomposition analysis. Telecommunications Policy 43, № 1, pp. 76-87.

Oaxaca, Ronald. 1973. Male-female wage differentials in urban labor markets. International economic review, pp. 693-709.

OECD. 2016. Skills Matter: Further Results from the Survey of Adult Skills. OECD Skills Studies. París: OECD Publishing.

Ono, Hiroshi y Madeline Zavodny. 2005. Gender differences in information technology usage: A US-Japan comparison. Sociological Perspectives 48, № 1, pp. 105-133

Pagés-Serra, Carmen y Claudia Piras. 2010. The Gender Dividend: Capitalizing on Women's Work. № 7531. Inter-American Development Bank.

Rashid, Ahmed Tareq. 2016. Digital inclusion and social inequality: Gender differences in ICT access and use in five developing countries. Gender, Technology and Development 20, № 3, pp. 306-332.

Sobaih, Abu Elnasr, Philip Coleman, Caroline Ritchie y Eleri Jones. 2011. Part-time restaurant employee perceptions of management practices: An empirical investigation. The Service Industries Journal 31, no. 11, pp. 1749-1768.

Van Laar, Ester, Alexander JAM Van Deursen, Jan AGM Van Dijk y Jos De Haan. 2017. The relation between 21st-century skills and digital skills: A systematic literature review. Computers in human behavior, 72, pp. 577-588

Wasserman, Ira M. y Marie Richmond-Abbott. 2005. Gender and the Internet: Causes of variation in access, level, and scope of use. Social science quarterly 86, no. 1, pp. 252-270

Weller, Jürgen, Sonia Gontero, and Susanna Campbell. 2019. Cambio tecnológico y empleo: una perspectiva latinoamericana. Riesgos de la sustitución tecnológica del trabajo humano y desafíos de la generación de nuevos puestos de trabajo. 


\title{
Sobre los autores
}

\author{
Hernán Galperín: University of Southern California. \\ Malena Arcidiacono: Universidad Nacional de La Plata (UNLP - Argentina)
}

\section{Notas}

El trabajo de la OCDE reporta diferencias de género en las habilidades digitales a favor de los hombres en Ecuador, Perú y México en función del porcentaje de hombres y mujeres que reprueban una prueba básica de habilidades TIC. Es

$\uparrow 1$ importante tener en cuenta que este indicador es una medición directa de las habilidades digitales, y no está basado en habilidades reportadas por los propios participantes, que tienden a favorecer a los hombres simplemente debido al sesgo de exceso de confianza.

Los ingresos no están incluidos en el cuestionario de México, pero la encuesta divide a los hogares en 4 niveles socioeconómicos basados en los activos del hogar y otros atributos. 